\title{
Phospholipid Encapsulated AuNR@Ag/Au Nanosphere SERS Tags with Environmental Stimulus Responsive Signal Property
}

\author{
Xueming Su, ${ }^{\dagger, \ddagger}$ Yunqing Wang, ${ }^{*}$, Wenhai Wang, Kaoxiang Sun, ${ }^{\ddagger}, \dagger$ and Lingxin Chen $*,+$ \\ ${ }^{\dagger}$ School of Pharmacy, Collaborative Innovation Center of Advanced Drug Delivery System and Biotech Drugs in Universities of \\ Shandong, Key Laboratory of Molecular Pharmacology and Drug Evaluation (Yantai University), Ministry of Education, Yantai \\ University, Yantai 264005, China \\ ${ }^{\ddagger}$ Key Laboratory of Coastal Environmental Processes and Ecological Remediation, Yantai Institute of Coastal Zone Research, Chinese \\ Academy of Sciences, Yantai 264003, China
}

\section{Supporting Information}

ABSTRACT: Surface-enhanced Raman scattering (SERS) tags draw much attention due to the ultrasensitivity and multiplex labeling capability. Recently, a new kind of SERS tags was rationally designed by encapsulating metal nanoparticles with phospholipid bilayers, showing great potential in theranostics. The lipid bilayer coating confers biocompatibility and versatility to changing surface chemistry of the tag; however, its "soft" feature may influence SERS signal stability, which is rarely investigated. Herein, we prepared phospholipidcoated AuNR@Ag/Au nanosphere SERS tags by using three different kinds of Raman reporters, i.e., thio-containing 4nitrothiophenol (NT), nitrogen-containing hydrophobic chro-

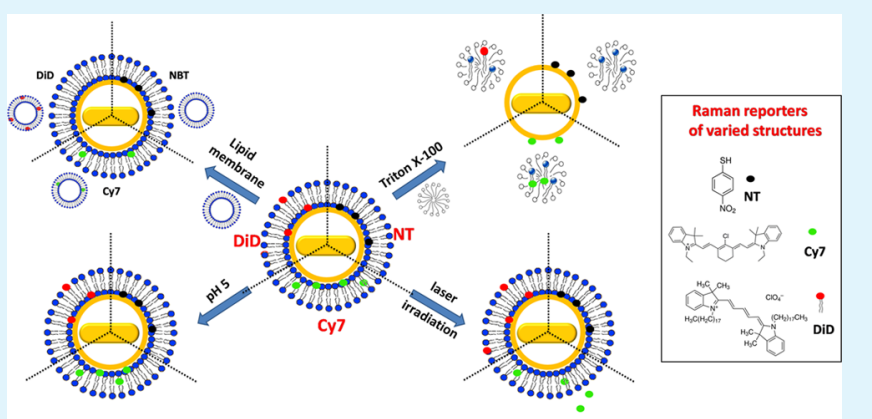

Phospholipid encapsulated, environmentally sensitive SERS tags mophore cyanine 7 monoacid (Cy7), and alkyl chainchromophore conjugate $1,1^{\prime}$-dioctadecyl-3,3,3',3'-tetramethylindodicarbocyanine (DiD). It was found that signal responses were different upon additional stimulation which the tags may encounter in theranostic applications including the presence of detergent Triton X-100, lipid membrane, and photothermal treatment. Living-cell imaging also showed signal changing distinction. The different SERS signal performances were attributed to the different Raman reporter releasing behaviors from the tags. This work revealed that Raman reporter structure determined signal stability of lipid-coated SERS tags, providing guidance for the design of stimulus responsive tags. Moreover, it also implied the potential of SERS technique for real time drug release study of lipid based nanomedicine.

KEYWORDS: surface-enhanced Raman scattering (SERS), gold nanoparticle, lipid bilayer, signal stability, cell imaging

\section{INTRODUCTION}

Surface-enhanced Raman scattering (SERS) imaging technique has shown great potential in biological analysis and diagnostic applications due to its sensitivity, high throughput recognition, and quantitative abilities. ${ }^{1-4}$ In the imaging platform, SERS tags are responsible for the generation of optical signal, which typically consist of noble metallic nanoparticles (NPs) as SERS substrates, specific organic Raman reporter molecules for producing characteristic scattering signals, and surface coating materials for NP stabilization. ${ }^{5-7}$

Gold NPs are the most widely used SERS substrates, which have many advantages in biological application including biocompatibility and long-term stability. However, their Raman signal-enhancing ability is not ideal and is 10 -fold to 100 -fold lower than those of similar silver nanostructures, restricting the ultrasensitive and in vivo imaging applications. To overcome this problem, many efforts were made on the development of novel gold plasmonic nanostructures with large field enhancement to increase the electromagnetic (EM) contribution for SERS. ${ }^{10}$ Recently, novel Au@Ag/Au NP was synthesized by converting the silver shell of a Au@Ag bimetallic $\mathrm{NP}$ into a porous $\mathrm{Ag} / \mathrm{Au}$ alloy layer via a galvanic replacement reaction, obtaining much enhanced SERS performance compared with that of the Au NP seed. ${ }^{11,12}$ On one hand, $\mathrm{Au} @ \mathrm{Ag} / \mathrm{Au}$ NPs usually have a strong, broad absorption crosssection, which is a benefit for matching the wavelength of the laser line and the absorption peak of Raman reporters for resonant signal enhancement. ${ }^{13,14}$ On the other hand, Au@Ag/ $\mathrm{Au}$ NPs exhibit a two-dimensional structure; there is a gap between the seed and the porous outer shell, rendering inherent EM hot spots. Raman reporters entering this gap feel a strong EM field and produce strong signals. ${ }^{12}$ Moreover, the additional high light-to-heat conversion feature further adds to their value in biomedical application, making them ideal candidates for the construction of multifunctional theranostic platforms. $^{15-17}$

Received: February 4, 2016

Accepted: April 6, 2016 
Surface modification materials are crucial for SERS tags for they impart stability and biocompatibility. Phospholipid bilayer is a promising noble metal NP coating ${ }^{18-26}$ and has been reported for the encapsulation of SERS tags. For example, Walker et al. prepared lipid encapsulated SERS tags incorporating MGITC, L-tryptophan, and rhodaminelissamine DSPE as Raman reporters. ${ }^{27}$ Tam et al. synthesized porphyrinphospholipid conjugates serving as both Raman reporters and surface coating agents on $\mathrm{Au}$ NPs, and the resultant tags are capable of cellular SERS imaging. ${ }^{28}$ Antibody-modified, lipidcoated SERS tags were also reported for in vitro chronic lymphocytic leukemia cells targeting analysis. ${ }^{29}$ These works demonstrated that the lipid coating could be facilely obtained via lipid self-assemble and it could render biocompatibility, colloidal stability, and even targeting capability of SERS tags.

However, it should be noted that this coating has its particularity because (1) lipid forms a "soft" coating and the structural integrity is not as rigid as the "solid" counterpart; ${ }^{30}$ (2) lipid provides additional encapsulation force to Raman reporters, which may alter their interaction with metal NP; and (3) Raman reporters with different chemical structures have varied interaction modes with lipid and thus they may encapsulate at different positions in lipid-coated SERS tags. All these factors hint that SERS performances of lipid-coated tags might be sensitive to environmental stimulus that can alter the structural integrity of lipid bilayers, such as temperature variation and the addition of detergent, due to the Raman reporter release. Up to now, the environment dependent SERS signal behaviors of lipid-coated tags have been rarely investigated.

In the present study, we prepared phospholipid-coated AuNR@Ag/Au nanosphere SERS tags by using three different kinds of Raman reporters, i.e., thio-containing small molecule 4nitrothiophenol (NT), nitrogen-containing hydrophobic chromophore cyanine 7 monoacid (Cy7), and alkyl chainchromophore conjugate 1,1'-dioctadecyl-3,3,3',3'-tetramethylindodicarbocyanine (DiD), for producing SERS signals, respectively. The signal stability of each tag was satisfactory in water and cell culture medium. However, the tags exhibited different signal responses in additional environmental stimulating conditions including the presence of detergent Triton X100 , excess lipid bilayer membrane, and photothermal treatment, determined by different Raman reporter releasing behaviors from tags. Living-cell imaging also showed the difference in signal variation with NT tag being the most stable while the signal of $\mathrm{DiD}$ tag disappeared fast. These results revealed that SERS performance of lipid-coated tags relied on dual interactions of reporters with both metal NP and lipid bilayer; thus the environmentally responsive SERS signal behaviors were more complicated than those of "bare" tags.

\section{EXPERIMENTAL SECTION}

Materials. Chloroauric acid $\left(\mathrm{HAuCl}_{4}\right)$, silver nitrate $\left(\mathrm{AgNO}_{3}\right)$, sodium borohydride $\left(\mathrm{NaBH}_{4}\right)$, sodium hydroxide $(\mathrm{NaOH})$, L-ascorbic acid (AA), crystal violet (CV), and phosphotungstic acid were purchased from Sinopharm Chemical Reagent Co., Ltd. Cetyltrimethylammonium bromide (CTAB), 1-naphthalenethiol (NAT), 4nitrothiophenol (NT), 3,3'-diethylthiatricarbocyanine iodide (DTTC), tris(2,2'-bipyridyl)ruthenium(II) chloride (Rubpy) were obtained from Sigma-Aldrich. 1,1' -Dioctadecyl-3,3,3',3'-tetramethylindodicarbocyanine (DiD) was obtained from Head (Beijing) Biotechnology Co., Ltd. Cyanine 7 monoacid (Cy7) was synthesized in our laboratory. ${ }^{31}$ Lipoid E 80 was purchased from Lipoid $\mathrm{GmbH}$ Germany. According to the manufacturer's specifications, it was comprised of $80 \%$ phosphatidylcholine, $8 \%$ phosphatidylethanolamine, $3.6 \%$ nonpolar lipids, and about $2 \%$ sphingomyelin. 3-(4,5Dimethylthiazol-2-yl)-2,5-diphenyltetrazolium bromide (MTT) was purchased from Thremo Scientific. LysoTracker DND-26 was obtained from Yeasen. Deionized water was used in all of the experiments.

Characterization. TEM images were acquired on a JEM-1400 transmission electron microscope (JEOL, Tokyo, Japan). SEM images and energy-dispersive X-ray spectra of the samples were obtained by using a field-emission microscope (Hitachi S-4800, Tokyo, Japan) equipped with an EX-350 energy-dispersive X-ray microanalyzer (HORIBA EMAX Energy). UV/vis/near-IR absorption spectra were recorded on a Thermo Scientific NanoDrop 2000/2000C spectrophotometer. FT-IR spectra were obtained from an FT/IR-4100 FT-IR spectrometer (JASCO, Tokyo, Japan). The hydrodynamic diameters were measured on a Zetasizer NanoZS90 (Malvern Instruments, Malvern, U.K.). The fluorescence spectra were measured on a FluoroMax-4 spectrofluorometer (HORIBA, Montpellier, France). The SERS spectra were recorded by using a DXR Raman Microscope (Thermo Scientific, Waltham, MA, USA). A $632.8 \mathrm{~nm}$ He:Ne laser was focused by a $10 \times$ and $50 \times$ microscope objective for sample solution and cell measurement, respectively, with a power of $1 \mathrm{~mW}$. The fluorescence images were captured using a Fluoview FV1000 confocal laser scanning microscope (Olympus, Tokyo, Japan) equipped with a $60 \times$ oil-immersion objective lens (NA 1.4): Cy7 and DiD channel, excitation (EX) of $635 \mathrm{~nm}$ and emission (EM) of $655-745 \mathrm{~nm}$; LysoTracker DND-26 channel, EX of $488 \mathrm{~nm}$ and EM of 505-535 nm.

Preparation of Gold Nanorods (AuNRs). AuNRs were synthesized by using the seed-mediated growth method. ${ }^{32}$ Briefly, the seed solution was prepared by reducing $\mathrm{HAuCl}_{4}(0.5 \mathrm{mM}, 2 \mathrm{~mL})$ in CTAB $(0.2 \mathrm{M}, 2 \mathrm{~mL})$ with freshly prepared ice-cold $\mathrm{NaBH}_{4}(10$ $\mathrm{mM}, 0.24 \mathrm{~mL}$ ). After $2 \mathrm{~h}, 3.6 \mathrm{~mL}$ of resultant seed solution was added into a growth solution of $\mathrm{HAuCl}_{4}(23 \mathrm{mM}, 13 \mathrm{~mL}), \mathrm{CTAB}(0.2 \mathrm{M}$, $200 \mathrm{~mL}), \mathrm{AgNO}_{3}(4 \mathrm{mM}, 11.2 \mathrm{~mL})$, and $\mathrm{AA}(80 \mathrm{mM}, 5 \mathrm{~mL})$. The mixture was left overnight at $27-30{ }^{\circ} \mathrm{C}$.

Preparation of AuNR@Ag NPs.AuNR@Ag NPs were synthesized according to a reported method with some minor modifications. ${ }^{15}$ The as-prepared AuNRs $(3 \mathrm{~mL})$ were centrifuged twice for 15 $\mathrm{min}$ at the speeds of 9200 and $8800 \mathrm{rpm}$, respectively. Then $10 \mathrm{~mL}$ of CTAB $(0.2 \mathrm{M})$ was added and followed by the addition of water until the total volume was $60 \mathrm{~mL}$. Then $600 \mu \mathrm{L}$ of AA $(0.1 \mathrm{M})$ and $3 \mathrm{~mL}$ of $\mathrm{AgNO}_{3}(10 \mathrm{mM})$ were added in sequence at room temperature under stirring. Finally, the $\mathrm{pH}$ of the mixture was adjusted to about 10 with addition of $\mathrm{NaOH}(0.1 \mathrm{M})$. The solution color immediately started to change from pink to orange, indicating the formation of a Ag layer on AuNR.

Preparation of AuNR@Ag/Au Nanosphere. The AuNR@Ag/ $\mathrm{Au}$ nanosphere was fabricated by galvanic replacement reaction with different volumes of $\mathrm{HAuCl}_{4}(1 \mathrm{mM})$. As-prepared AuNR@Ag NP solution $(10 \mathrm{~mL})$ was mixed with $10 \mathrm{~mL}$ of CTAB solution $(0.1 \mathrm{M})$ in a $50 \mathrm{~mL}$ glass tube. Then $\mathrm{HAuCl}_{4}$ at different volumes of solution was injected into the mixture at a rate of $100 \mu \mathrm{L} / \mathrm{min}$ under stirring at 100 ${ }^{\circ} \mathrm{C}$. The mixture was allowed to react for $15 \mathrm{~min}$, and then the sample was centrifuged $(6600 \mathrm{rpm}, 15 \mathrm{~min})$ and concentrated 10 -fold for further use.

Preparation of Lipid-Coated SERS Tags with One Kind of Reporter. The lipid-coated SERS tags were prepared using a conventional thin film hydration method. ${ }^{28}$ Briefly, the lipid was dissolved in ethanol $(5 \mathrm{mg} / \mathrm{mL})$ followed by the addition of Raman reporter molecules NT $\left(10^{-3} \mathrm{M}, 100 \mu \mathrm{L}\right), \mathrm{DiD}\left(7.94 \times 10^{-4} \mathrm{M}, 5 \mu \mathrm{L}\right)$, or Cy7 $\left(4.62 \times 10^{-3} \mathrm{M}, 5 \mu \mathrm{L}\right)$, respectively. The solvent of the mixture was evaporated at $37{ }^{\circ} \mathrm{C}$ under vacuum in a rotary evaporator, and the obtained lipid film was placed under vacuum for $1 \mathrm{~h}$ to ensure the complete drying. Then the film was hydrated with $1 \mathrm{~mL}$ of AuNR@ $\mathrm{Ag} / \mathrm{Au}$ nanosphere solution. Each sample was then subjected to three rounds of vortexing $(30 \mathrm{~s})$, heating to $85^{\circ} \mathrm{C}(45 \mathrm{~s})$, and sonication $(30$ s) until all precipitates were dissolved into the solution. Then the sample was left overnight at about $23^{\circ} \mathrm{C}$. The lipid-coated SERS tags were purified by centrifugation at $6600 \mathrm{rpm}$ for $15 \mathrm{~min}$ for three 
rounds. Excess lipids in the supernatant were removed, and the tags were resuspended in water for further analysis.

Preparation of Lipid-Coated SERS Tags with Three Kinds of Reporters. First, AuNR@Ag/Au nanosphere was labeled with NT by adding $100 \mu \mathrm{L}$ of NT solution $\left(5 \times 10^{-4} \mathrm{M}\right)$ into $1 \mathrm{~mL}$ of AuNR@Ag/ $\mathrm{Au}$ nanosphere solution. After incubation for $1 \mathrm{~h}$, the mixture was centrifuged for $15 \mathrm{~min}$ at $6600 \mathrm{rpm}$ to remove the excess NT. Then liposomes containing both $\mathrm{Cy} 7$ and $\mathrm{DiD}$ reporters were prepared. Dried lipid film was formed by evaporating $2 \mathrm{~mL}$ of lipid ethanol solution $(5 \mathrm{mg} / \mathrm{mL})$ with Cy7 $\left(4.62 \times 10^{-6} \mathrm{M}, 5 \mu \mathrm{L}\right)$ and $\mathrm{DiD}(1.59$ $\times 10^{-6} \mathrm{M}, 10 \mu \mathrm{L}$ ) onto the side of a round-bottom flask using a rotary evaporator, followed by further drying under vacuum overnight. The film was then hydrated in $2 \mathrm{~mL}$ of water at $40{ }^{\circ} \mathrm{C}$ for $1 \mathrm{~h}$. Periodic vortexing of the sample suspended the lipid film into solution, and then sonication was performed with a $3 \mathrm{~mm}$ probe tip at a power of $100 \mathrm{~W}$. Sonication was performed in $100 \mathrm{~s}$ cycles with $60 \mathrm{~s}$ resting and cooling between each cycle for a total of $12 \mathrm{~min}$ at room temperature. Then NT labeled NP solution was mixed with the liposome solution in equal volume, followed by vortexing and standing for overnight. The mixture was centrifuged and stored in $4{ }^{\circ} \mathrm{C}$ refrigerator for further use.

Preparation of Small Unilamelar Vesicles. Small unilamelar vesicles (SUVs) were prepared as liposome acceptors in a liposomeSERS tag lipid exchange experiment. Dried lipid film was prepared by evaporating $5 \mathrm{~mL}$ of ethanol $(5 \mathrm{mg} / \mathrm{mL})$ solution onto the sides of a round-bottom flask using a rotary evaporator, followed by further drying under vacuum overnight to remove traces of the organic solvent. The film was then hydrated in $5 \mathrm{~mL}$ of water at $40{ }^{\circ} \mathrm{C}$ for $1 \mathrm{~h}$. Periodic vortexing of the sample suspended the lipid film into solution, and then sonication was performed with a $3 \mathrm{~mm}$ probe tip at a power of $100 \mathrm{~W}$. Sonication was performed in $100 \mathrm{~s}$ cycles with $60 \mathrm{~s}$ resting and cooling between each cycle for a total of $12 \mathrm{~min}$ at room temperature. The translucent solution was then centrifuged for $20 \mathrm{~min}$ at $12000 \mathrm{rpm}$, and the supernatant was filtered through a $0.22 \mu \mathrm{m}$ filter and then diluted to a volume of $10 \mathrm{~mL}$. The SUVs had an average hydrodynamic diameter of $59 \mathrm{~nm}$ by dynamic light scattering measurement.

Photothermal Treatment. The samples were filled in a $1.5 \mathrm{~mL}$ Eppendorf tube and irradiated by a near-IR laser $(785 \mathrm{~nm}, 400 \mathrm{~mW})$ at a distance of $1 \mathrm{~cm}$ from the side of the solution. A thermoindicator was used to monitor the temperature change. The temperature and Raman signal of each sample were measured at different intervals.

Fluorescence Measurement. The quantity of Raman reporter detached from the lipid-coated tags during the stimulus was measured by fluorescence. The samples with and without irradiation treatment were centrifuged at a speed of $6600 \mathrm{rpm}$ for $15 \mathrm{~min}$. The recovered precipitates were dispersed in $500 \mu \mathrm{L}$ of ethanol, followed by sonication to resolve the remaining $\mathrm{Cy} 7$ or $\mathrm{DiD}$ in the tags. Then the mixtures were centrifuged, and the supernatants were subjected to fluorescence measurement. For Triton X-100 dissolution and liposome fusion experiments, the tag solution was mixed with Triton X-100 or liposome solution, and then centrifuged at a speed of $6600 \mathrm{rpm}$ for 15 min. The supernatant was collected for fluorescence measurement. The excitation wavelengths of Cy7 and $\mathrm{DiD}$ were 658 and $644 \mathrm{~nm}$, respectively.

Cell Culture. SMMC7721 cells (a liver-cancer cell line) were grown as a monolayer in a humidified incubator at $37{ }^{\circ} \mathrm{C}$ in air $/ \mathrm{CO}_{2}$ (95:5) in an RPMI-1640 medium that was supplemented with $10 \%$ fetal bovine serum. For all experiments, the cells were harvested by using trypsin and were resuspended in fresh medium before plating.

MTT Assay. In a 96-well plate, $8 \times 10^{3}$ SMMC7721 cells were plated and incubated for $12 \mathrm{~h}$ to allow the cells to attach. SERS tag solution was added to the cells to form NP suspensions in culture media with different concentrations. Then the cells were incubated at $37^{\circ} \mathrm{C}$ for another $24 \mathrm{~h}$. Cell viability of the tags was evaluated by using an MTT assay.

Cell Imaging. SMMC7721 cells $\left(2 \times 10^{4}\right)$ were seeded onto glass coverslips in a 24 -well plate with $500 \mu \mathrm{L}$ of culture medium to allow the cells to attach. Then the cells were cultured for $12 \mathrm{~h}$ for cells to attach. Then $100 \mu \mathrm{L}$ of each tag was added into the cells. After incubation for 2,4 , and $8 \mathrm{~h}$, the cell monolayer on the coverslip was observed with Raman microscopy and the SERS signals could be measured at the same time.

Live-Cell Fluorescence Colocalization Study. The attached SMMC7721 cells were incubated with SERS tags $(210 \mu \mathrm{g} / \mathrm{mL}, 300$ $\mu \mathrm{L})$ for $2 \mathrm{~h}$. The culture medium was discarded, and the cells were washed with phosphate-buffered saline (PBS). To stain lysosomes, the cells were further incubated with LysoTracker Green DND-26 (150 $\mathrm{nM}, 300 \mu \mathrm{L}$ ) for $30 \mathrm{~min}$. Then the cells were washed with PBS twice for further fluorescence colocalization imaging.

\section{RESULTS AND DISCUSSION}

Preparation of AuNR@Ag/Au Nanosphere.AuNR@Ag/ $\mathrm{Au}$ nanospheres as SERS substrates were prepared via a galvanic replacement reaction. ${ }^{15}$ AuNR seed was first prepared with about a $10 \mathrm{~nm}$ width and a $40 \mathrm{~nm}$ length, for which the transverse and longitudinal SPR peaks are shown centered at 520 and $780 \mathrm{~nm}$, respectively (Figure 1A). Subsequently, the

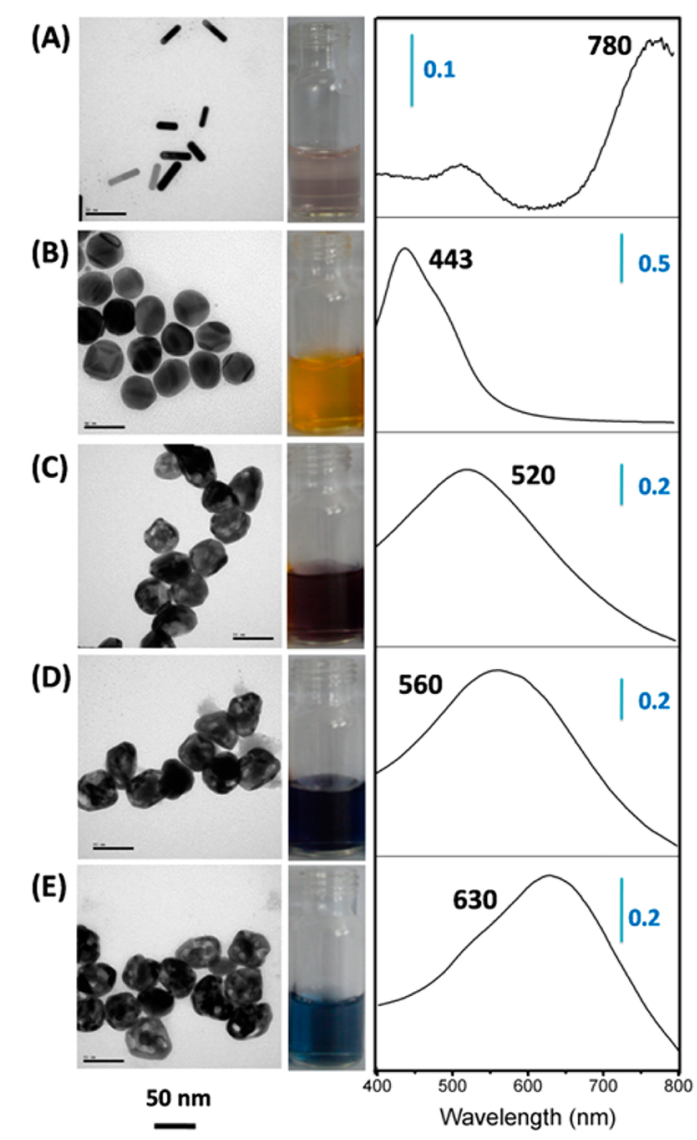

Figure 1. Transmission electron microscopy (TEM) image, photographs of solution, and UV-vis spectra of of AuNR seed (A), AuNR@ Ag NPs (B), and AuNR@Ag/Au nanosphere prepared by adding 1, 1.5 , and $2 \mathrm{~mL}$ of $1 \mathrm{mM} \mathrm{HAuCl}_{4}$ solution $(\mathrm{C}-\mathrm{E})$, respectively. UV-vis spectra were recorded at the same NP concentration.

Ag layer was deposited on AuNR resulting in the formation of sphere-like AuNR@Ag bimetallic NPs. It could be clearly seen that AuNR was embedded in the AuNR@Ag NP with a diameter of around $48 \mathrm{~nm}$ with a clear differential contrast (Figure 1B). The corresponding absorption spectrum revealed that the longitudinal SPR band of AuNR vanished and a strong peak at $443 \mathrm{~nm}$ emerged, resulting from the transverse dipole resonance of electrons at the thick side of the Ag shell. Panels $\mathrm{C}-\mathrm{E}$ of Figure 1 illustrated TEM images, photograph images, 
and UV-vis absorption spectra of AuNR@Ag/Au nanospheres prepared by adding different amounts of $\mathrm{HAuCl}_{4}$ to AuNR@Ag NPs. After the replacement reaction, the yolk-shell structure with $\mathrm{AuNR}$ core and $\mathrm{Ag} / \mathrm{Au}$ alloy shell was clearly observed, and the overall shapes and sizes of NPs did not obviously change compared with AuNR@Ag NPs. With more $\mathrm{HAuCl}_{4}$ added, increasing gaps could be observed and the colors of the resultant products varied from yellow to purple to blue. UV-vis absorption spectra showed a red shift from 520 to $630 \mathrm{~nm}$ with much broader bands and stronger optical intensity as more galvanic replacement reaction occurred.

The elemental composition variation of the NP before and after galvanic replacement reaction was characterized by energy-dispersive X-ray spectra. As shown in Supporting Information Figure $\mathrm{S} 1$, the silver-gold content (atom percentage) ratio of AuNR@Ag NP was 76:17. After the addition with $1.5 \mathrm{~mL}$ of $\mathrm{HAuCl}_{4}$, the ratio changed to about 25:66; the increasing content of Au element further confirmed the occurrence of galvanic replacement reaction. Considering the structural integrity and the optical property, AuNR@Ag/Au nanosphere prepared by adding $2 \mathrm{~mL}$ of $\mathrm{HAuCl}_{4}$ was used in the following experiments.

SERS Feature of AuNR@Ag/Au Nanosphere. The Raman enhancing ability of AuNR@Ag/Au nanosphere was further investigated by recording SERS spectra of four kinds of Raman reporters of varied absorption peak positions (NAT, CV, Rubpy, and DTTC) in NP solution, comparing with those from AuNR solution of the same concentration. As illustrated in Figure 2, the characteristic Raman peaks could be observed for all reporters from AuNR@Ag/Au nanosphere, among which $\mathrm{CV}$ and DTTC showed extremely high intensities. By contrast,

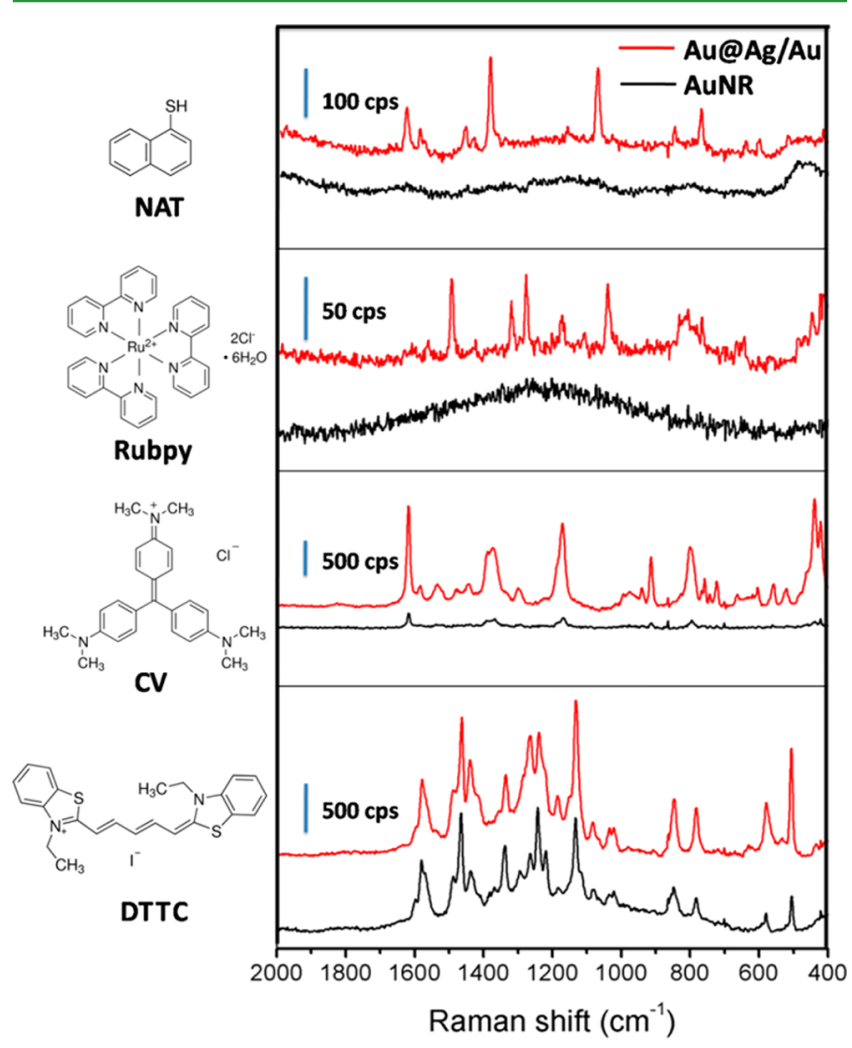

Figure 2. SERS spectra of NAT $\left(10^{-4} \mathrm{M}\right), \mathrm{CV}\left(10^{-6} \mathrm{M}\right)$, Rubpy $\left(10^{-5}\right.$ $\mathrm{M})$, and DTTC $\left(10^{-5} \mathrm{M}\right)$ in AuNR@Ag/Au nanosphere and AuNR colloids of the same concentration. when AuNR was applied as substrate, only DTTC showed strong SERS signal comparable with that from AuNR@Ag/Au nanosphere, and those of the other three reporters were much weaker or even undetectable. The ideal enhancing ability of AuNR@Ag/Au nanosphere might be attributed to the following two reasons. First, the plasmon resonance is advantageous to cover a broader region of the electromagnetic spectrum from visible to near-IR region, which perfectly match not only the excitation-laser wavelength but also the absorption peaks of various kinds of Raman reporters. This surfaceenhanced resonant Raman scattering effect is a benefit for producing a higher enhancement factor. ${ }^{33,34}$ For AuNR, the longitude SPR peak at $780 \mathrm{~nm}$ matched the maximum absorption of DTTC $(764 \mathrm{~nm})$; thus only DTTC exhibited strong Raman intensity. Second, the coupling between the $\mathrm{AuNR}$ core and the external porous $\mathrm{Ag} / \mathrm{Au}$ shell renders the inherent EM hot spots. Raman reporters may enter the shell through diffusion and reach these hot spots, achieving a further signal enhancement. ${ }^{12}$ The versatile and sensitive sensing ability of AuNR@Ag/Au nanosphere was crucial for the subsequent study on lipid-coated SERS tags that need to label different kinds of Raman reporters.

Preparation of Lipid-Coated SERS Tags. Subsequently, the lipid encapsulation was performed followed the procedures shown in Figure 3A. Raman reporters were mixed with phospholipids in ethanol and dried as a lipid film under $\mathrm{N}_{2}$ gas in a round-bottom flask. The lipid film was directly hydrated with AuNR@Ag/Au nanosphere colloid, followed by vortexing, heating, and sonication to ensure uniform phospholipid coating on a AuNR@Ag/Au nanosphere surface. Such treatments were essential to dissolve and disperse the dried lipid layer to form small liposomes. Thereafter the liposomes with large surface area efficiently took part in the molecular exchange process with CTAB on AuNR@Ag/Au nanosphere. The molecular exchange occurred because cationic surfactant CTAB was electrostatically attracted to the AuNR@ $\mathrm{Ag} / \mathrm{Au}$ nanospheres, intermediated by bromide ions bonding to the NP surface and the trimethylammonium headgroup. The headgroup of the lipid phosphatidylcholine terminates with a similar quaternary ammonium cation, so that the lipid could also be electrostatically attracted to NPs with comparable affinity with CTAB. ${ }^{35}$ When CTAB-coated NPs were mixed with liposomes, CTAB would be displaced by lipid. The molecular displacement could reach essentially complete when a large excess of phospholipid was present. ${ }^{18,36}$ The lipid exchange could also be promoted by vortexing and sonication treatments.

SEM images (Figure 3B,C) showed that the surrounding of bare AuNR@Ag/Au nanosphere was clean whereas diffuse coronas appeared around the lipid bilayer coated tags. TEM characterization of the lipid-coated NPs was also performed by negative staining with $1 \%$ phosphotungstic acid for increasing the contrast. As shown in Figure 3D, white coatings around NPs with the thickness of about $5 \mathrm{~nm}$ could be distinguished, indicating the formation of a lipid layer. FT-IR spectra (Figure 3E) revealed that the characteristic peaks of phospholipid including carbonyl $\nu(\mathrm{C}=\mathrm{O})\left(1735 \mathrm{~cm}^{-1}\right)$, phosphate $\nu_{\mathrm{a}}\left(\mathrm{PO}_{2}^{-}\right)\left(1238 \mathrm{~cm}^{-1}\right.$ at the shoulder $)$, and $\nu \mathrm{s}(\mathrm{C}-\mathrm{O}-\mathrm{C})$ $\left(1087 \mathrm{~cm}^{-1}\right)$ were readily observable in lipid-coated AuNR@ $\mathrm{Ag} / \mathrm{Au}$ nanosphere. ${ }^{18,37}$ For bare NPs, only weak peaks of alkyl chains appeared originating from a tiny amount of remaining CTAB ligand. The hydrodynamic diameter of the lipid-coated tags, compared with that of the bare counterparts was measured 
(A)
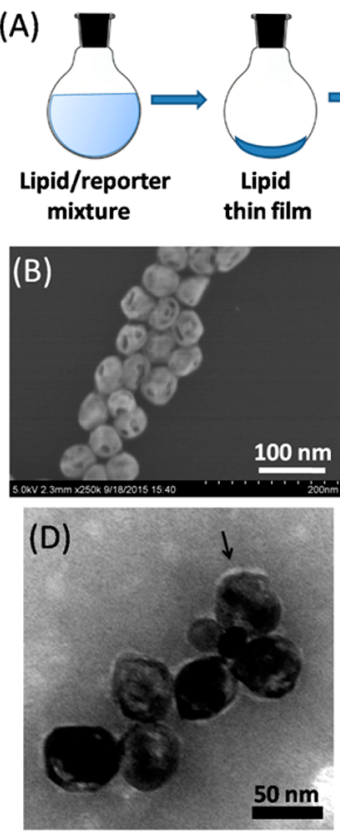

(F)

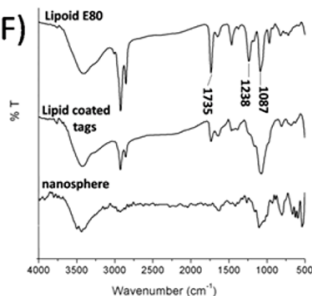

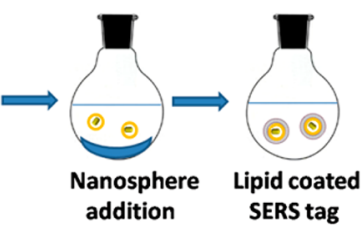

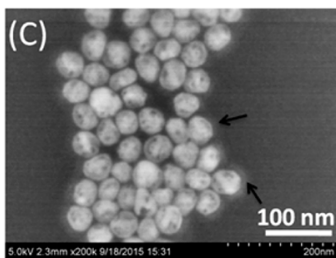

(E)

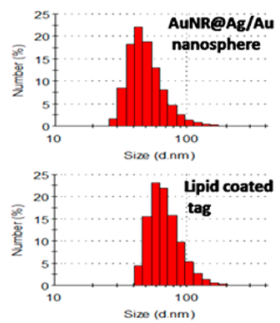

(G)

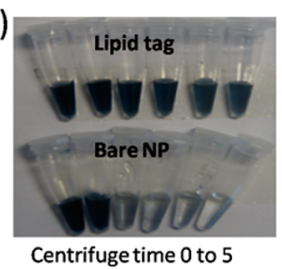

Figure 3. (A) Schematic illustration (not to scale) of the method to produce lipid-coated SERS tags. Panels B and C show SEM images of bare AuNR@Ag/Au nanosphere and lipid-coated SERS tags, respectively. Black arrows indicate the diffuse coronas around AuNR@Ag/Au nanospheres. (D) TEM of lipid-coated SERS tags. The sample was negatively stained by $1 \%$ phosphotungstic acid solution. Black arrow indicates the lipid coating around AuNR@Ag/ Au nanospheres. (E) Hydrodynamic diameter distributions of AuNR@ $\mathrm{Ag} / \mathrm{Au}$ nanosphere and the lipid-coated tags. (F) FT-IR spectra of lipoid E80, lipid-coated SERS tag, and bare AuNR@Ag/Au nanosphere. (G) Dispersity of lipid-coated SERS tag and bare AuNR@Ag/ $\mathrm{Au}$ nanosphere after centrifugation and resuspension for different times.

by dynamic light scattering (DLS) method. The diameter increased from $52 \mathrm{~nm}$ (with polydispersity index of 0.28 ) to around $71 \mathrm{~nm}$ (with polydispersity index of 0.29 ) because of the presence of the lipid bilayer (Figure 2F). It should be noted that DLS measured the hydrodynamic diameter of NPs, thus illustrating a larger size than that from SEM or TEM characterizations. The increase of the colloidal stability of NPs also indicated the lipid bilayer coating. The lipid-coated tags could be dispersed even after five centrifugation and resuspension steps whereas AuNR@Ag/Au nanospheres aggregated and totally lost optical absorption after a mere two rounds of treatment (Figure 3G).

During the lipid-coating process, three hydrophobic reporters of $\mathrm{NT}, \mathrm{Cy} 7$, and $\mathrm{DiD}$ were selected as Raman reporters to produce characteristic SERS signals because they contained different featured functional groups (i.e.,-SH, - $\mathrm{N}$, or long alkyl chain) and had specific but distinct interaction modes (i.e., chemi- or physi-adsorption or hydrophobic intercalation) with metallic surface and lipid bilayer. As illustrated by the

schematic diagram in Figure 4, different Raman reporters might be stabilized at different binding sites in the tags by different dominant interactions, depending on the chemical structures. It was speculated that NT had a high affinity to metal and was likely to attach on the AuNR@Ag/Au nanosphere surface via a $\mathrm{Au}-\mathrm{S}$ bond. In the case of $\mathrm{Cy} 7$, a portion of the reporters was intercalated into the double hydrocarbon chains of lipid via hydrophobic interaction during the sonication process. ${ }^{38}$ The possibility of adsorption to the metal for the portion that reached the NP surface also could not be ruled out. ${ }^{27}$ By contrast, as a lipophilic cyanine dye which is specific for fluorescent labeling cytoplasmic membrane or liposome, DiD could directly insert into the lipid bilayer via its two long 18carbon chains induced strong hydrophobic interactions, leaving dye headgroups at both ends of the lipid bilayer. ${ }^{39,40}$ As shown in Figure 4C-E, each purified lipid-coated SERS tag solution illustrated a strong and characteristic SERS spectrum of the corresponding Raman reporter, confirming that all kinds of reporters were closely associated with the AuNR@Ag/Au nanosphere because of the short length scale over which the SERS effect could occur. ${ }^{27,41}$

The SERS intensity variations of each tag under different environmental stimuli were further investigated. First, Triton X100 , a detergent that can form micelle in water and dissolve the lipid bilayer, was added to tag solutions. ${ }^{42}$ After Triton X-100 treatment, the hydrodynamic diameter of the tags decreased from around 70 to $54 \mathrm{~nm}$, which was similar to the size of bare NPs (Figure S2). TEM images also revealed that the white lipid shell around the tags disappeared (Figure S3). Furthermore, SERS intensity changes were monitored after the lipid dissociation, which were helpful for understanding the interaction mode and spatial distribution of Raman reporter in each tag. As shown in Figure 4C, the intensity of NT tag remained stable, proving the stable attachment of NT on the AuNR@Ag/Au nanosphere surface. The SERS signal of the Cy7 tag decreased to about half of the initial value (Figure 4D), indicating a part of $\mathrm{Cy} 7$ remained on the metal and the portion entrapped in lipid bilayer was released. By contrast, the SERS signal of $\mathrm{DiD}$ tag totally disappeared as a result of the complete DiD transferring from tags to Triton X-100 micelles, together with the lipid coatings of the tags (Figure 4E). Fluorescence characterization results were consistent with those from SERS measurements. Both $\mathrm{Cy} 7$ and $\mathrm{DiD}$ fluorescence in supernatant appeared after mixing with Triton X-100 as a result of the dye release (Figure S4). These results indicated that DiD was mainly immobilized by the hydrophobic force with lipid chains in the tags but not the $\mathrm{N}-\mathrm{Au}$ interaction from the cyanine dye headgroup.

Considering the outstanding photothermal feature of AuNR@Ag/Au nanospheres (Figure S5A), and the potential hyperthermia treatment application of lipid-coating SERS tags, the signal stability encountered $785 \mathrm{~nm}$ near-IR laser irradiation was studied. Blank experiments revealed that no obvious change in Raman intensities was observed after 7 days at $4{ }^{\circ} \mathrm{C}$ storage, indicating satisfactory long-term stability for all samples. However, as shown in Figure 5A, after laser irradiation for $20 \mathrm{~min}$ at $400 \mathrm{~mW}$ power, different tags showed varied SERS intensity changing performances. The signals of both NT and $\mathrm{DiD}$ tags were nearly stable. However, the intensity of $\mathrm{Cy} 7$ tag dramatically decreased to about $29 \%$ of its initial value.

During the irradiation process, the temperatures of tag solutions increased from $22{ }^{\circ} \mathrm{C}$ to around $33^{\circ} \mathrm{C}$ and reached equilibrium (Figure S5B). All three samples almost maintained 

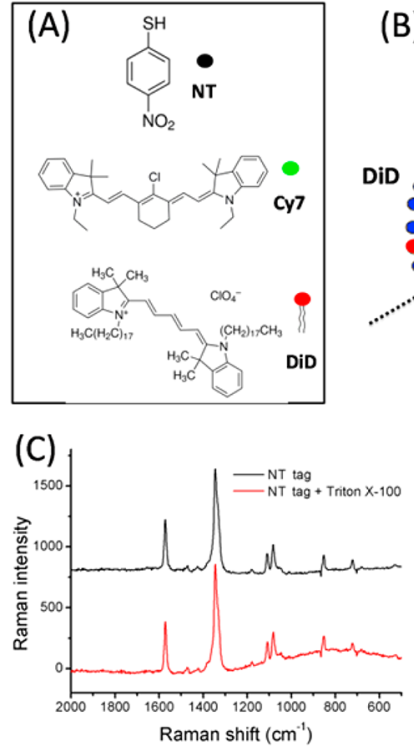
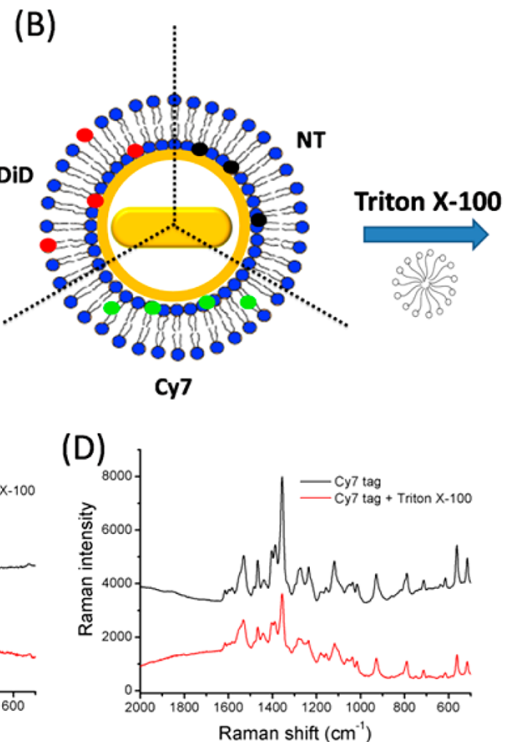

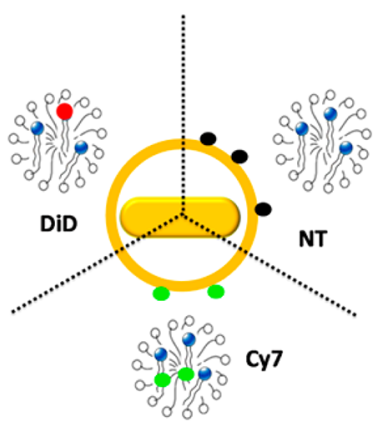

$(\mathrm{E})$

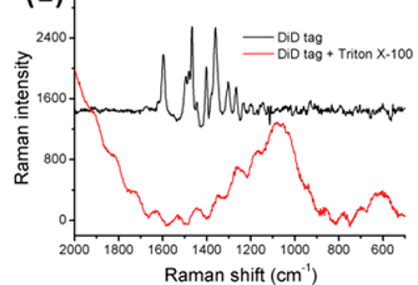

Figure 4. (A) Chemical structure of three Raman reporters of NT, Cy7, and DiD. (B) Schematic illustration of the location of each Raman reporter in lipid-coated tags and the lipid dissolution after the addition of $10 \%$ Triton X-100. For concise illustrating, three Raman reporter labeling conditions were shown in one particle with three divided parts, separated by cutoff rules. Each part represents one kind of tag. Panels $\mathrm{C}-\mathrm{E}$ represent SERS spectra of lipid-coated NT tag, Cy7 tag, and DiD tag before and after adding Triton X-100, respectively.
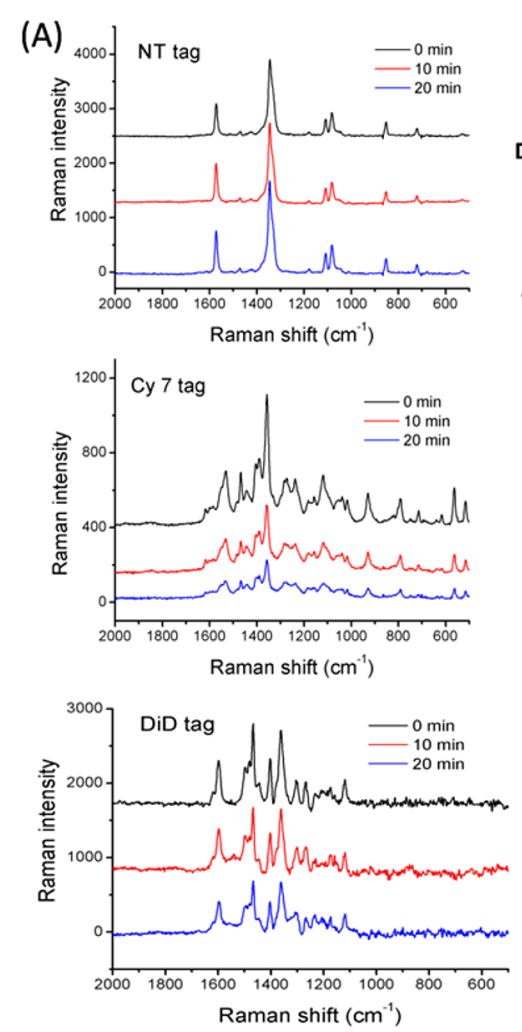
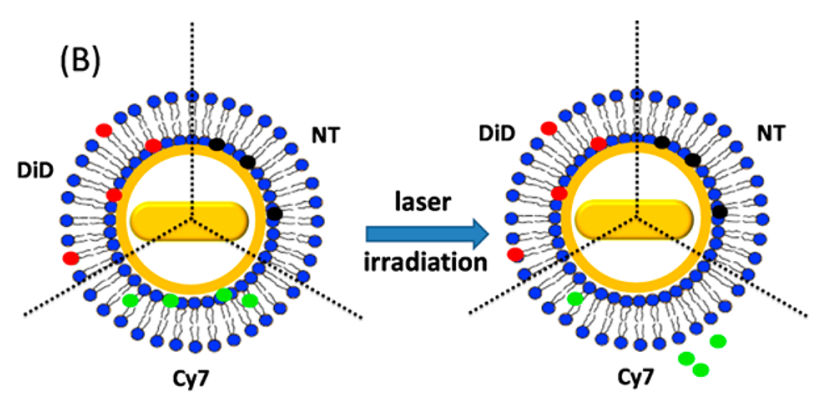

(C)
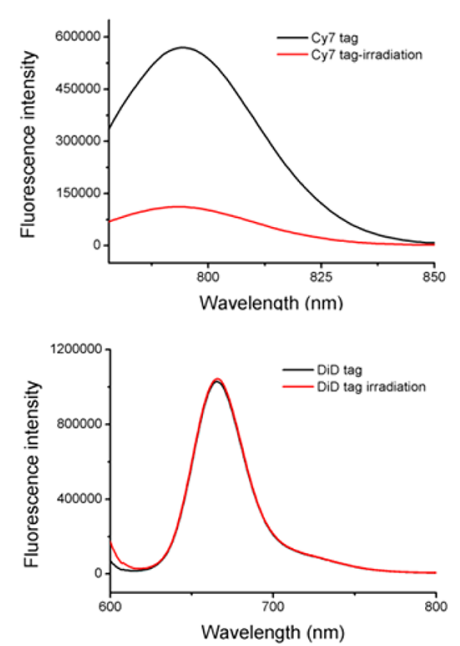

Figure 5. (A) SERS spectra of different tags before and after laser irradiation for 10 and $20 \mathrm{~min}$. (B) Schematic illustration of the location of each Raman reporter in lipid-coated tags after photothermal treatment. For concise illustrating, three Raman reporter labeling conditions were shown in one particle with three divided parts, separated by cutoff rules. Each part represents one kind of tag. (C) Fluorescence spectra of Cy7 and DiD in SERS tags before and after laser irradiation.

their UV-vis absorbances in this period, indicating the monodispersity of the tags. Therefore, the decrease of SERS intensity should be attributed to the loss of Raman reporters induced by photothermal effect. In a NP-lipid bilayer system, the influence factors were complicated because heating might affect interactions of reporter with both metal and lipid, as well as the structural stability of the lipid bilayer. To make it simplified, the irradiation effect on a sole AuNR@Ag/Au nanosphere-reporter complex without lipid coating was first studied. As shown in Figure S6, the signal of the Cy7-AuNR@ 

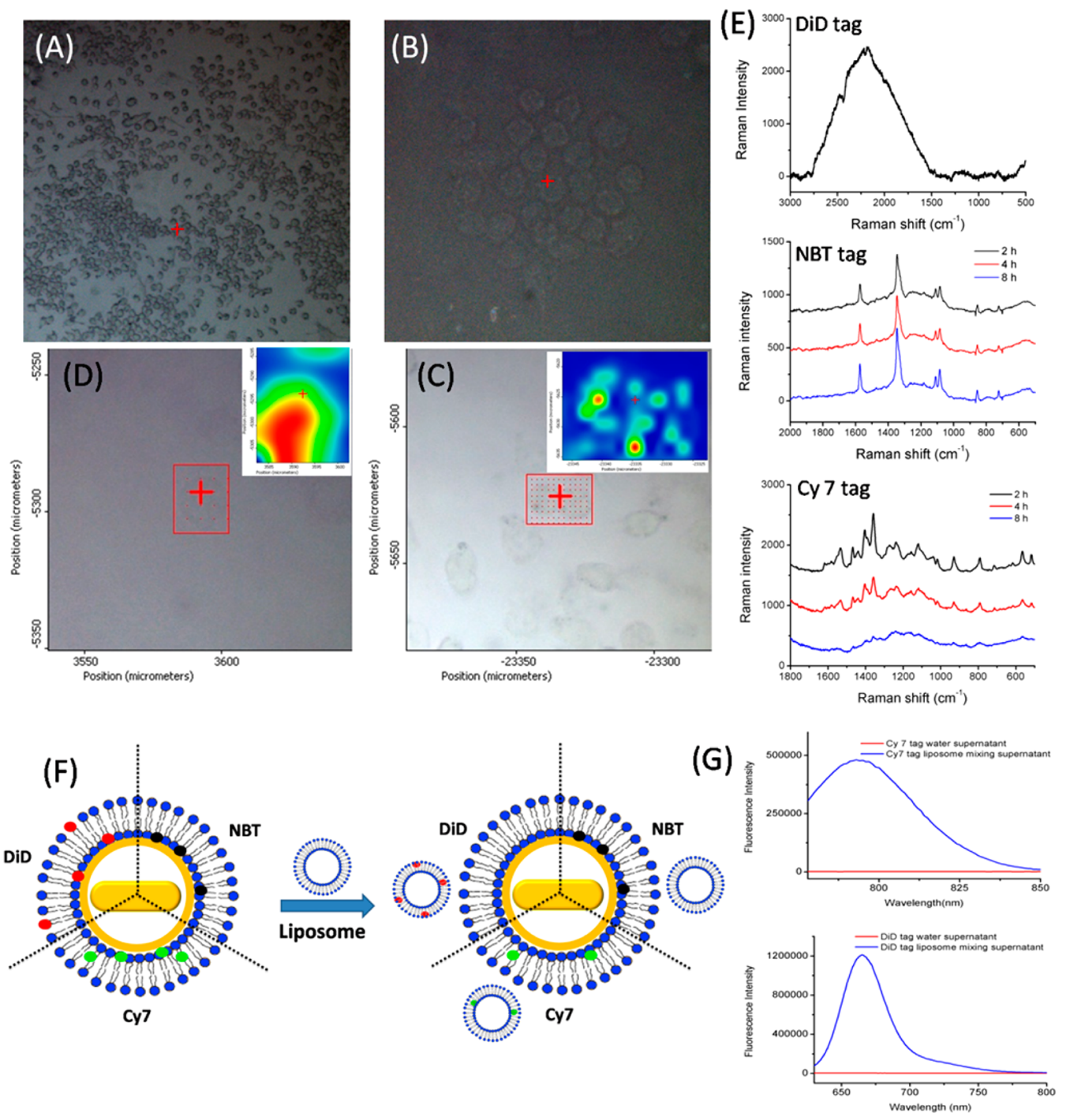

Figure 6. (A) Bright- and (B) dark-field images of SMMC7721 cells after incubating Cy7 SERS tags for 2 h. The images were acquired with a $\times 10$ and a $\times 50$ objective lens, respectively. Panels C and D represent Raman mapping of NT tag and Cy7 tag in a single SMMC7721 cell, respectively, after incubating for $2 \mathrm{~h}$. The brighter sites indicate more tags based on the ratio of the peak intensities at $1344 \mathrm{~cm}^{-1}$ for NT tag and $1356 \mathrm{~cm}^{-1}$ for Cy7 tag. (E) SERS spectra obtained from DiD tag labeled cell after $2 \mathrm{~h}$ incubation and from NT tag and Cy7 tag labeled cells after 2, 4, and $8 \mathrm{~h}$ incubation. (F) Schematic illustration of the location of each Raman reporter in lipid-coated tags after liposome fusion. For concise illustrating, three Raman reporter labeling conditions were shown in one particle with three divided parts, separated by cutoff rules. Each part represents one kind of tag. (G) Fluorescence spectra of the supernatants of $\mathrm{Cy} 7$ tag-liposome and $\mathrm{DiD}$ tag-liposome mixtures. A control experiment was performed by mixing the tags with water, and the supernatant showed no fluorescence for each tag.

$\mathrm{Ag} / \mathrm{Au}$ nanosphere complex decreased sharply, indicating the laser could break the electrostatic interaction between metal NP and reporter. This was similar to that physi-adsorbed drug doxorubicin could release from gold nanoshell surface under photothermal treatment, ${ }^{43}$ whereas the $\mathrm{Au}-\mathrm{S}$ bond was much stronger and the signal of NT-AuNR@Ag/Au nanosphere was not affected as expected. The stable DiD Raman signal indicated the retention of $\mathrm{DiD}$ in the $\mathrm{CTAB}$ layer of the AuNR@Ag/Au nanosphere.

For lipid-coating tags, additional lipid-Raman reporter interaction should also be taken into account. It was reported that heating could lead to a phase transition of lipid bilayer from solid gel phase to liquid disordered phase, ${ }^{44}$ resulting in the packing defects and increasing permeability of lipid bilayer for both hydrophilic and hydrophobic molecules. ${ }^{20,44}$ As shown in Figure 5B, a number of $\mathrm{Cy} 7$ molecules detached from $\mathrm{AuNR} \mathrm{Ag} / \mathrm{Au}$ nanosphere under irradiation, penetrated the leaking liquid phase lipid bilayer, and then released to the solution. By contrast, as a structural analogue to the natural lipid, the intercalated DiD would not affect the phospholipid assembles process and the integrity of the bilayer structure around NPs. Photothermal effect induced heating caused a lipid bilayer phase change but not the loss of lipid. Thus, the compact bilayer could still "lock in" $\mathrm{DiD}$ and the SERS intensity was nearly stable.

To further provide evidence for this reporter release induced SERS intensity changing, the amount of Raman reporter in lipid-coated tags with and without laser irradiation was analyzed by fluorescence spectra. The treated $\mathrm{Cy} 7$ and $\mathrm{DiD}$ tags, and blank controls, were centrifuged, and the precipitates were redispersed in ethanol to dissolve the remaining reporters. Then the fluorescence of each reporter released in ethanol supernatant was measured. As shown in Figure 5C, the fluorescence of $\mathrm{Cy} 7$ acquired from photothermal treated sample was much 

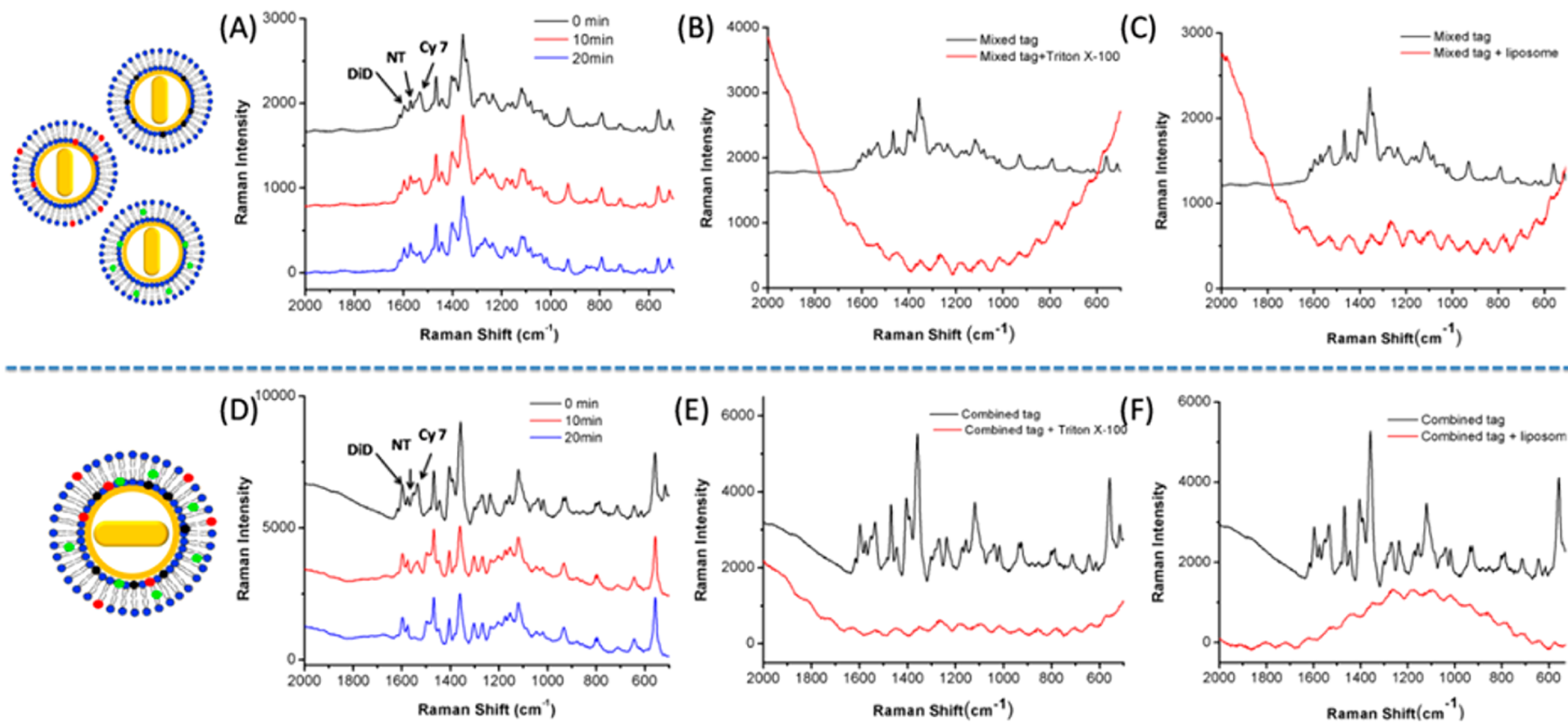

Figure 7. Upper panel: schematic illustration of the mixing of lipid-coated tags with a single kind of reporter, and the SERS spectra before and after photothermal treatment (A), addition of Triton X-100 (B), and addition of liposome (C). Lower panel: schematic illustration of the lipid-coated tags labeling with three kinds of reporters simultaneously, and the SERS spectra before and after photothermal treatment (D), addition of Triton X-100 (E), and addition of liposome (F).

lower than that from the untreated one, and the fluorescence of DiD was almost stable after laser irradiation, which was consistence with the above SERS results.

Live-Cell Imaging. The cytotoxicity of the lipid-coated tags was estimated by MTT assays on SMMC7721 cells (a livercancer cell line). The MTT results indicated that there were no significant decreases in cell viability after incubation at a dose of $1.0 \mu \mathrm{g} \mathrm{mL}^{-1}$ tags (Figure S7), showing the feasibility of the tags for cell imaging. To examine the intracellular SERS signal response, SMMC7721 cells were incubated with each tag. Bright- and dark-field optical imaging of live cells was employed to locate the live cells. It could be observed that NPs were distributed in cytoplasmic regions after $2 \mathrm{~h}$ of incubation, indicating the uptake of the NPs by cells (Figure 6A,B). Simultaneously, SERS measurements were performed using a $632.8 \mathrm{~nm}$ laser with a power of $\sim 1 \mathrm{~mW}$. Panels $\mathrm{C}$ and D of Figure 6 showed that strong and characteristic SERS signals of NT and Cy7 tags could be detected from a single cell, respectively. By contrast, no SERS signal was observable in DiD tag labeled cells except for a broad fluorescence peak, which was quite different from the result of the other two tags (Figure $6 \mathrm{E})$.

It was reported that during cell incubation, NPs passed through cytomembrane and intracellular membranes ${ }^{45}$ at the stage of cell surface entering and endocytosis. Finally, they were trapped in the endosome and then transported to lysosome encountering an acidic environment with $\mathrm{pH}$ about $5 .^{46,47}$ In our case, the location of lipid-coated tags was verified by fluorescence colocalization using green fluorescence of LysoTracker DND-26 that could specifically stain the lysosome and the red fluorescence of $\mathrm{Cy} 7$ or $\mathrm{DiD}$ dye in the tags. As shown in Figure S8, images in columns a-d were bright-field images, tag fluorescence images, LysoTracker Green DND-26 fluorescence images, and the merged images, respectively. In the merged images, yellow color appeared in cells, indicating the colocalization of the tracker and tags in lysosomes.
In vitro experiments showed that the $\mathrm{pH}$ change of $\mathrm{DiD}$ tag solution from 7.4 to 5.0 did not cause apparent SERS intensity variation (Figure S9). Previous reports revealed that lipid could transfer between vesicles and hybrid bilayers on $\mathrm{Au}$ nanoshells; ${ }^{48}$ therefore, there appeared to be an exchange of the lipid on the tags with the cellular membranes which would result in the loss of $\mathrm{DiD}$ and vanishing of SERS signals. This possibility was tested by a liposome fusion experiment. Small unilamelar vesicles, as analogues of cells or a subcellular organelle of lipid bilayer membrances, were prepared as lipid acceptors and mixed with each tag as a lipid donor (Figure 6F). As shown in Figure S10, the signal of the NT tag remained stable and the signal of the $\mathrm{Cy} 7$ tag slightly decreased while that of $\mathrm{DiD}$ tag totally disappeared, leaving a high random background. Furthermore, fluorescence measurements were performed on the supernatants of $\mathrm{Cy} 7 \mathrm{tag}$-liposome and $\mathrm{DiD}$ tag-liposome mixtures. As shown in Figure 6G, the fluorescence of $\mathrm{Cy} 7$ or $\mathrm{DiD}$ appeared in supernatant after liposome fusion, indicating the transferring of dyes from SERS tag to blank liposome. A control experiment was performed by mixing the tags with equal volumes of water, followed by centrifugation and fluorescence measurement. The supernatant showed no fluorescence for each tag. These results implied the release of dyes in a lipid membrane-rich environment, providing evidence for the lipid exchange between tags and cellular membranes in living cells.

Time dependent SERS signals were also monitored from NT tag and Cy7 tag labeled cells. As shown in Figure 6E, in $8 \mathrm{~h}$ incubation period, the SERS intensity of NT tag was pretty stable whereas a dramatic signal decrease was observed for Cy7 tag, which was probably ascribed to gradual detachment of Cy7 from tags induced by the competitive replacement of endogenous small thiol molecules such as glutathione. ${ }^{49,50}$

The discovery of the stimulus responsive signal property of a single type reporter labeled tag encouraged us to perform more challenging experiments with all three signals presented for each of the stimuli. Two mixing modes were tested. The first 
was that three kinds of tags (each tag was labeled with one kind of Raman reporter) were mixed, and each stimulus was applied to the mixing solution. As shown in Figure $7 \mathrm{~A}-\mathrm{C}$, in the mixing SERS spectra, characteristic peaks of each Raman reporter could be distinguished and were sensitive to changing of environmental conditions. The second was that all three kinds of Raman reporters were simultaneously labeled on one NP to form a "combined" tag. Then this tag was subject to each stimulus. As shown in Figure $7 \mathrm{D}-\mathrm{F}$, a characteristic peak of each Raman reporter also appeared, and the SERS profile changing feature upon stimulus was similar to that from the single reporter labeled tag. Under photothermal treatment, the peak of Cy7 gradually decreased with the peaks of NT and DiD unchanged (Figure 7D). When mixing with Triton X-100 (Figure 7E) or liposome (Figure 7F), the SERS signal of the "combined" tag was submerged in fluorescence as a result of transferring of $\mathrm{Cy} 7$ and DiD dyes from NPs to Triton X-100 micelles or liposomes. These results indicated the possibility of lipid-coated tags in developing a multiresponsive sensor device based on SERS signal measurements.

Overall, the SERS signal intensity of a SERS tag $P^{\text {SERS }}$ can be described by the following equation:

$$
P^{\mathrm{SERS}}\left(\nu_{\mathrm{S}}\right)=N \sigma_{\mathrm{ads}}^{\mathrm{R}}\left|A\left(\nu_{\mathrm{L}}\right)\right|^{2}\left|A\left(\nu_{\mathrm{S}}\right)\right|^{2} I\left(\nu_{\mathrm{L}}\right)
$$

Here, $I\left(\nu_{\mathrm{L}}\right)$ is the excitation-laser intensity; $\sigma_{\mathrm{ads}}^{\mathrm{R}}$ is the Raman cross-section of the adsorbed molecule; $N$ is the number of molecules that undergo the SERS process; and $A\left(\nu_{\mathrm{L}}\right)$ and $A\left(\nu_{\mathrm{S}}\right)$ are laser and Raman scattering field enhancement factors, respectively. ${ }^{51}$ From this equation, one could see the distances of the SERS substrate and reporters, the surface densities of the reporters, and the dispersing state of the tags, as a reviewer indicated. Experimental results revealed that the tag was monodispersed in each test condition. Therefore, the SERS intensity of the lipid-coated tag was mainly affected by the former two factors, which were both fundamentally determined by the attaching stability of Raman reporter in SERS tags. Reporters with different chemical structures have different interaction modes with NP substrate and lipid layer, thus illustrating varied detaching behaviors under stimulus and the resultant different SERS signal performances.

\section{CONCLUSION}

We herein demonstrated preparation and environmentally related signal stability of phospholipid-coated AuNR@Ag/Au nanosphere SERS tags with different kinds of Raman reporters. In the lipid-coated tags, the retention of Raman reporters relied on dual interactions with metal surface and lipid bilayer; thus the SERS signal changing behaviors were more complicated than that from "bare" tags. It was found that the NT tag was the most stable in all tested conditions due to strong binding between the metal and thiol group. The signal of the Cy7 tag decreased gradually under photothermal treatment and in living cells as a result of the detachment of Cy7 from the AuNR@Ag/ $\mathrm{Au}$ nanosphere surface. By contrast, the SERS intensity of the $\mathrm{DiD}$ tag was very sensitive in detergent or bilayer membranerich environments because of the loss of $\mathrm{DiD}$ reporter during lipid dissolution or exchange process. Our work revealed the Raman reporter structure determined, environmental responsive SERS performance of lipid-coated tags, which not only provided guidance for the synthesis of novel SERS tags, but also implied the feasibility of real time SERS monitoring drug release in lipid based theranostic nanoplatforms.

\section{ASSOCIATED CONTENT}

\section{S Supporting Information}

The Supporting Information is available free of charge on the ACS Publications website at DOI: 10.1021/acsami.6b01523.

EDX spectra of AuNR@Ag NPs and AuNR@Ag/Au nanosphere, DLS analysis results of lipid-coated tags before and after different environmental stimulus, TEM images of lipid-coated SERS tags without and with Triton X-100 treatment, heating curves of AuNR@Ag/ Au nanosphere and lipid-coated SERS tags, SERS spectra of bare AuNR@Ag/Au nanosphere reporter under 785 $\mathrm{nm}$ laser irradiation, cytotoxicity assay of the lipid-coated SERS tags, fluorescence spectra of the supernatants of tags after each stimulus, confocal fluorescence colocalization results of lipid-coated tags in living cells, SERS spectra of lipid-coated Cy7 tag and DiD tag in different $\mathrm{pH}$ conditions, SERS spectra of each lipid-coated tag before and after the addition of small unilamelar vesicles (PDF)

\section{AUTHOR INFORMATION}

\section{Corresponding Authors}

*E-mail: yqwang@yic.ac.cn. Fax: +86 535 2109130. Tel.: +86 5352109130 (Y.Q.W.).

*E-mail: sunkx@ytu.edu.cn. Fax: +86 535 6706066. Tel.: +86 5353808266 (K.X.S.).

*E-mail: lxchen@yic.ac.cn. Fax: +86 535 2109130. Tel.: +86 5352109130 (L.X.C.).

\section{Notes}

The authors declare no competing financial interest.

\section{ACKNOWLEDGMENTS}

Financial support from the National Natural Science Foundation of China (Grants 81573393, 21305157, 81102415 , and 21575159), the 12th Five Years Key Programs for Science and Technology Development of China (Grant 2013ZX09402201), the Natural Science Foundation of Shandong (Grants ZR2014HM062 and ZR2013HQ009), and the Science and Technology Development Plan of Shandong Province of China (Grant 2014GGF01074) is gratefully acknowledged.

\section{REFERENCES}

(1) Schlucker, S. Surface-Enhanced Raman Spectroscopy: Concepts and Chemical Applications. Angew. Chem., Int. Ed. 2014, 53, 47564795.

(2) Ling, X.; Huang, S.; Deng, S.; Mao, N.; Kong, J.; Dresselhaus, M. S.; Zhang, J. Lighting up the Raman Signal of Molecules in the Vicinity of Graphene Related Materials. Acc. Chem. Res. 2015, 48, 1862-1870.

(3) Abramczyk, H.; Brozek-Pluska, B. Raman Imaging in Biochemical and Biomedical Applications. Diagnosis and Treatment of Breast Cancer. Chem. Rev. 2013, 113, 5766-5781.

(4) Lane, L. A.; Qian, X.; Nie, S. SERS Nanoparticles in Medicine: From Label-Free Detection to Spectroscopic Tagging. Chem. Rev. 2015, 115, 10489-10529.

(5) Wang, Y.; Yan, B.; Chen, L. SERS Tags: Novel Optical Nanoprobes for Bioanalysis. Chem. Rev. 2013, 113, 1391-1428.

(6) Xie, W.; Schlucker, S. Medical Applications of Surface-Enhanced Raman Scattering. Phys. Chem. Chem. Phys. 2013, 15, 5329-5344.

(7) Zhang, W.; Wang, Y.; Sun, X.; Wang, W.; Chen, L. Mesoporous Titania Based Yolk-Shell Nanoparticles as Multifunctional Theranostic Platforms for SERS Imaging and Chemo-Photothermal Treatment. Nanoscale 2014, 6, 14514-14522. 
(8) Saha, K.; Agasti, S. S.; Kim, C.; Li, X.; Rotello, V. M. Gold Nanoparticles in Chemical and Biological Sensing. Chem. Rev. 2012, 112, 2739-2779.

(9) Abalde-Cela, S.; Aldeanueva-Potel, P.; Mateo-Mateo, C.; Rodriguez-Lorenzo, L.; Alvarez-Puebla, R. A.; Liz-Marzan, L. M. Surface-Enhanced Raman Scattering Biomedical Applications of Plasmonic Colloidal Particles. J. R. Soc., Interface 2010, 7, S435-S450.

(10) Vo-Dinh, T.; Liu, Y.; Fales, A. M.; Ngo, H.; Wang, H. N.; Register, J. K.; Yuan, H.; Norton, S. J.; Griffin, G. D. SERS Nanosensors and Nanoreporters: Golden Opportunities in Biomedical Applications. Wiley Interdiscip. Rev. Nanomed. Nanobiotechnol. 2015, 7, $17-33$.

(11) Xiong, W.; Mazid, R.; Yap, L. W.; Li, X.; Cheng, W. Plasmonic Caged Gold Nanorods for near-Infrared Light Controlled Drug Delivery. Nanoscale 2014, 6, 14388-14393.

(12) Jaiswal, A.; Tian, L.; Tadepalli, S.; Liu, K. K.; Fei, M.; Farrell, M. E.; Pellegrino, P. M.; Singamaneni, S. Plasmonic nanorattles with Intrinsic Electromagnetic Hot-Spots for Surface Enhanced Raman Scattering. Small 2014, 10, 4287-4292.

(13) McLintock, A.; Cunha-Matos, C. A.; Zagnoni, M.; Millington, O. R; Wark, A. W. Universal Surface-Enhanced Raman Tags: Individual Nanorods for Measurements from the Visible to the Infrared (514-1064 Nm). ACS Nano 2014, 8, 8600-8609.

(14) Garcia-Leis, A.; Torreggiani, A.; Garcia-Ramos, J. V.; SanchezCortes, S. Hollow Au/Ag Nanostars Displaying Broad Plasmonic Resonance and High Surface-Enhanced Raman Sensitivity. Nanoscale 2015, 7, 13629-13637.

(15) Ye, X. S.; Shi, H.; He, X. X.; Wang, K. M.; Li, D.; Qiu, P. C. Gold Nanorod-Seeded Synthesis of $\mathrm{Au} @ \mathrm{Ag} / \mathrm{Au}$ Nanospheres with Broad and Intense near-Infrared Absorption for Photothermal Cancer Therapy. J. Mater. Chem. B 2014, 2, 3667-3673.

(16) Gandra, N.; Portz, C.; Singamaneni, S. Multifunctional Plasmonic AuNR@Ag/Au nanospheres for Spectrum-Guided Locoregional Therapy. Adv. Mater. 2014, 26, 424-429.

(17) Shi, H.; Ye, X.; He, X.; Wang, K.; Cui, W.; He, D.; Li, D.; Jia, X. $\mathrm{Au} @ \mathrm{Ag} / \mathrm{Au}$ Nanoparticles Assembled with Activatable Aptamer Probes as Smart "Nano-Doctors" for Image-Guided Cancer Thermotherapy. Nanoscale 2014, 6, 8754-8761.

(18) Orendorff, C. J.; Alam, T. M.; Sasaki, D. Y.; Bunker, B. C.; Voigt, J. A. Phospholipid-Gold Nanorod Composites. ACS Nano 2009, 3, 971-983.

(19) Stewart, A. F.; Lee, A.; Ahmed, A.; Ip, S.; Kumacheva, E.; Walker, G. C. Rational Design for the Controlled Aggregation of Gold Nanorods Via Phospholipid Encapsulation for Enhanced Raman Scattering. ACS Nano 2014, 8, 5462-5467.

(20) Troutier, A. L.; Ladaviere, C. An Overview of Lipid Membrane Supported by Colloidal Particles. Adv. Colloid Interface Sci. 2007, 133, $1-21$.

(21) Kang, J. H.; Ko, Y. T. Lipid-Coated Gold Nanocomposites for Enhanced Cancer Therapy. Int. J. Nanomed. 2015, 10, 33-45.

(22) An, H. H.; Han, W. B.; Kim, Y.; Kim, H.-S.; Oh, Y.; Yoon, C. S. Preparation of SERS Active Ag Nanoparticles Encapsulated by Phospholipids. J. Raman Spectrosc. 2014, 45, 292-298.

(23) Yang, J. A.; Murphy, C. J. Evidence for Patchy Lipid Layers on Gold Nanoparticle Surfaces. Langmuir 2012, 28, 5404-5416.

(24) Rhim, W. K.; Kim, J. S.; Nam, J. M. Lipid-Gold-Nanoparticle Hybrid-Based Gene Delivery. Small 2008, 4, 1651-1655.

(25) Tam, N. C. M.; Scott, B. M. T.; Voicu, D.; Wilson, B. C.; Zheng, G. Facile Synthesis of Raman Active Phospholipid Gold Nanoparticles. Bioconjugate Chem. 2010, 21, 2178-2182.

(26) Lee, S. E.; Sasaki, D. Y.; Perroud, T. D.; Yoo, D.; Patel, K. D.; Lee, L. P. Biologically Functional Cationic Phospholipid-Gold Nanoplasmonic Carriers of RNA. J. Am. Chem. Soc. 2009, 131, 14066-14074.

(27) Ip, S.; MacLaughlin, C. M.; Gunari, N.; Walker, G. C. Phospholipid Membrane Encapsulation of Nanoparticles for SurfaceEnhanced Raman Scattering. Langmuir 2011, 27, 7024-7033.

(28) Tam, N. C.; McVeigh, P. Z.; MacDonald, T. D.; Farhadi, A.; Wilson, B. C.; Zheng, G. Porphyrin-Lipid Stabilized Gold Nano- particles for Surface Enhanced Raman Scattering Based Imaging. Bioconjugate Chem. 2012, 23, 1726-1730.

(29) Ip, S. Y.; MacLaughlin, C. M.; Mullaithilaga, N.; Joseph, M.; Wala, S.; Wang, C.; Walker, G. C. Lipid-Encapsulation of Surface Enhanced Raman Scattering (SERS) Nanoparticles and Targeting to Chronic Lymphocytic Leukemia (CLL) Cells. Proc. SPIE 2012, 8212, 821204.

(30) Al-Jamal, W. T.; Kostarelos, K. Liposome-Nanoparticle Hybrids for Multimodal Diagnostic and Therapeutic Applications. Nanomedicine 2007, 2, 85-98.

(31) Wang, R.; Chen, L.; Liu, P.; Zhang, Q.; Wang, Y. Sensitive nearInfrared Fluorescent Probes for Thiols Based on Se-N Bond Cleavage: Imaging in Living Cells and Tissues. Chem. - Eur. J. 2012, 18, 1134311349 .

(32) Nikoobakht, B.; El-Sayed, M. A. Preparation and Growth Mechanism of Gold Nanorods (Nrs) Using Seed-Mediated Growth Method. Chem. Mater. 2003, 15, 1957-1962.

(33) McNay, G.; Eustace, D.; Smith, W. E.; Faulds, K.; Graham, D. Surface-Enhanced Raman Scattering (SERS) and Surface-Enhanced Resonance Raman Scattering (SERRS): A Review of Applications. Appl. Spectrosc. 2011, 65, 825-837.

(34) Niu, X.; Chen, H.; Wang, Y.; Wang, W.; Sun, X.; Chen, L. Upconversion Fluorescence-SERS Dual-Mode Tags for Cellular and in Vivo Imaging. ACS Appl. Mater. Interfaces 2014, 6, 5152-5160.

(35) Matthews, J. R.; Payne, C. M.; Hafner, J. H. Analysis of Phospholipid Bilayers on Gold Nanorods by Plasmon Resonance Sensing and Surface-Enhanced Raman Scattering. Langmuir 2015, 31, 9893-9900.

(36) Takahashi, H.; Niidome, Y.; Niidome, T.; Kaneko, K.; Kawasaki, H.; Yamada, S. Modification of Gold Nanorods Using Phospatidylcholine to Reduce Cytotoxicity. Langmuir 2006, 22, 2-5.

(37) Pawlikowska-Pawlega, B.; Misiak, L. E.; Zarzyka, B.; Paduch, R.; Gawron, A.; Gruszecki, W. I. Localization and Interaction of Genistein with Model Membranes Formed with Dipalmitoylphosphatidylcholine (DPPC). Biochim. Biophys. Acta, Biomembr. 2012, 1818, 1785-1793.

(38) Levin, C. S.; Kundu, J.; Janesko, B. G.; Scuseria, G. E.; Raphael, R. M.; Halas, N. J. Interactions of Ibuprofen with Hybrid Lipid Bilayers Probed by Complementary Surface-Enhanced Vibrational Spectroscopies. J. Phys. Chem. B 2008, 112, 14168-14175.

(39) Yoon, T. Y.; Okumus, B.; Zhang, F.; Shin, Y. K.; Ha, T. Multiple Intermediates in Snare-Induced Membrane Fusion. Proc. Natl. Acad. Sci. U. S. A. 2006, 103, 19731-19736.

(40) Heberle, F. A.; Buboltz, J. T.; Stringer, D.; Feigenson, G. W. Fluorescence Methods to Detect Phase Boundaries in Lipid Bilayer Mixtures. Biochim. Biophys. Acta, Mol. Cell Res. 2005, 1746, 186-192.

(41) Lin, M.; Wang, Y.; Sun, X.; Wang, W.; Chen, L. "Elastic" Property of Mesoporous Silica Shell: For Dynamic Surface Enhanced Raman Scattering Ability Monitoring of Growing Noble Metal Nanostructures Via a Simplified Spatially Confined Growth Method. ACS Appl. Mater. Interfaces 2015, 7, 7516-7525.

(42) Lopez, O.; Cocera, M.; Pons, R.; Azemar, N.; Lopez-Iglesias, C.; Wehrli, E.; Parra, J. L.; de la Maza, A. Use of a Dynamic Light Scattering Technique to Study the Kinetics of Liposome Solubilization by Triton X-100. Langmuir 1999, 15, 4678-4681.

(43) You, J.; Zhang, G.; Li, C. Exceptionally High Payload of Doxorubicin in Hollow Gold Nanospheres for near-Infrared LightTriggered Drug Release. ACS Nano 2010, 4, 1033-1041.

(44) Kneidl, B.; Peller, M.; Winter, G.; Lindner, L. H.; Hossann, M. Thermosensitive Liposomal Drug Delivery Systems: State of the Art Review. Int. J. Nanomed. 2014, 9, 4387-4398.

(45) Wang, M.; Petersen, N. O. Characterization of Phospholipidcoated Gold Nanoparticles: A Versatile Platform to Study Drug Delivery and Cellular Uptake Mechanisms. Can. J. Chem. 2015, 93, 265-271.

(46) Ashley, C. E.; Carnes, E. C.; Phillips, G. K.; Padilla, D.; Durfee, P. N.; Brown, P. A.; Hanna, T. N.; Liu, J.; Phillips, B.; Carter, M. B.; Carroll, N. J.; Jiang, X.; Dunphy, D. R.; Willman, C. L.; Petsev, D. N.; Evans, D. G.; Parikh, A. N.; Chackerian, B.; Wharton, W.; Peabody, D. S.; Brinker, C. J. The Targeted Delivery of Multicomponent Cargos to 
Cancer Cells by Nanoporous Particle-Supported Lipid Bilayers. Nat. Mater. 2011, 10, 389-397.

(47) Hu, F.; Zhang, Y.; Chen, G.; Li, C.; Wang, Q. Double-Walled $\mathrm{Au}$ Nanocage $/ \mathrm{SiO}_{2}$ Nanorattles: Integrating SERS Imaging, Drug Delivery and Photothermal Therapy. Small 2015, 11, 985-993.

(48) Kundu, J.; Levin, C. S.; Halas, N. J. Real-Time Monitoring of Lipid Transfer between Vesicles and Hybrid Bilayers on $\mathrm{Au}$ Nanoshells Using Surface Enhanced Raman Scattering (SERS). Nanoscale 2009, 1, 114-117.

(49) Wang, Y.; Chen, L.; Liu, P. Biocompatible Triplex Ag@SiO ${ }_{2} @$ $\mathrm{mTiO}_{2}$ Core-Shell Nanoparticles for Simultaneous Fluorescence-SERS Bimodal Imaging and Drug Delivery. Chem. - Eur. J. 2012, 18, 59355943.

(50) Zong, S.; Wang, Z.; Chen, H.; Yang, J.; Cui, Y. Surface Enhanced Raman Scattering Traceable and Glutathione Responsive Nanocarrier for the Intracellular Drug Delivery. Anal. Chem. 2013, 85, 2223-2230.

(51) Kneipp, K.; Kneipp, H.; Kneipp, J. Surface-Enhanced Raman Scattering in Local Optical Fields of Silver and Gold NanoaggregatesFrom Single-Molecule Raman Spectroscopy to Ultrasensitive Probing in Live Cells. Acc. Chem. Res. 2006, 39, 443-450. 